\title{
Gallium fights infection in phase I trial
}

Currently available antibacterials — which target DNA replication, protein synthesis and cell wall biosynthesis - are becoming less and less effective owing to the increasing prevalence of antibiotic resistance. Strategies to target alternative cellular processes, such as bacterial nutrition and metabolism, are therefore gaining attention. Now, Singh and colleagues report that gallium, which disrupts iron-dependent processes, exhibits antibiotic activity in a mouse model of Pseudomonas aeruginosa airway infection and improves lung function in patients with cystic fibrosis (CF) and chronic $P$. aeruginosa lung infection in a preliminary phase I clinical trial.

Iron is essential for most bacteria as it is required in enzymes that mediate many key processes such as DNA synthesis, electron transport and oxidative stress defence. Given that some bacterial uptake systems are unable to distinguish between gallium and iron, gallium can substitute for iron and therefore disrupt iron-dependent processes. Previous studies have demonstrated that gallium compounds exert antibacterial

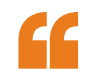

This study highlights the potential of therapeutically targeting nutritional vulnerabilities of bacterial pathogens 5

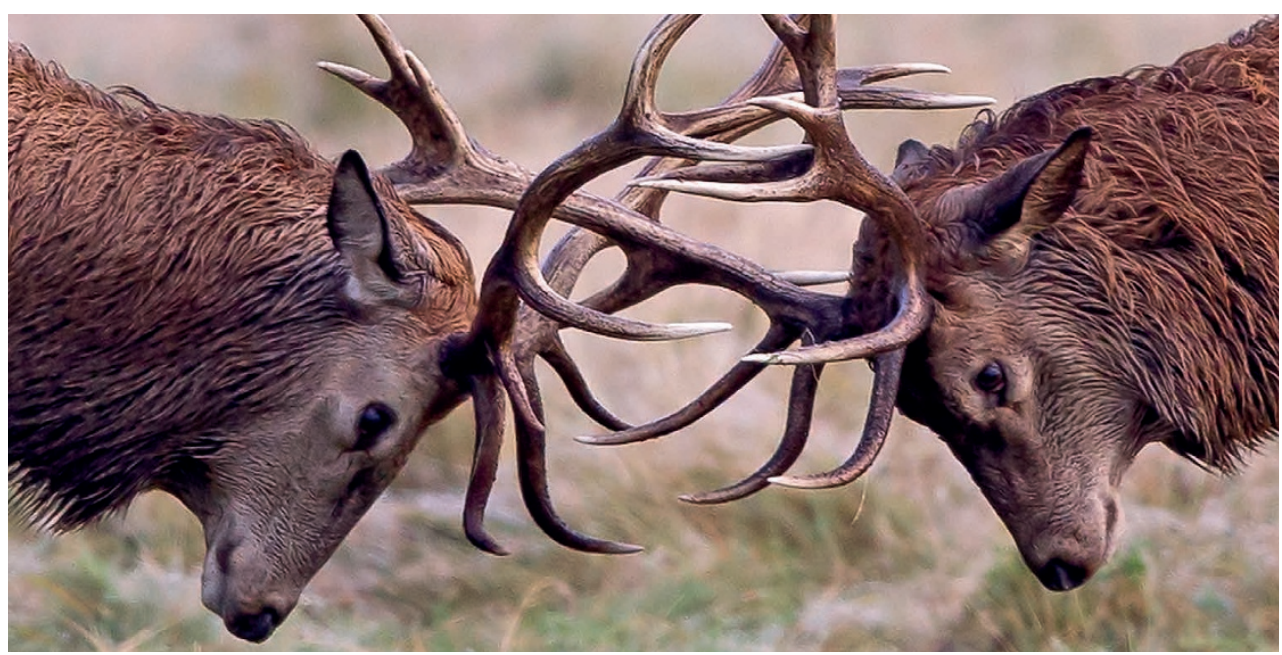

Chris Upson/Moment/Getty Images

activity against a number of human pathogens, including multidrugresistant CF clinical isolates. Singh and colleagues therefore set out to further test the potential of gallium as an antibacterial treatment.

First, the authors demonstrated that iron is a growth-limiting nutrient for $P$. aeruginosa in sputum from patients with CF. Micromolar concentrations of gallium inhibited $P$. aeruginosa growth in human CF sputum, an effect that was enhanced in iron-limiting conditions. Mechanistically, gallium was shown to inhibit iron-containing enzymes in $P$. aeruginosa in vitro, including activity of ribonucleotide reductase and catalase, and increase oxidant sensitivity.

Next, the authors assessed the potential of $P$. aeruginosa to develop resistance to gallium. In vitro, the rate of development of spontaneous resistance to gallium was low, with resistance to the exisiting antibiotics colistin, ciprofloxacin and tobramycin occurring about two times more frequently. Furthermore, the rate at which mutations arose under selection with gallium increased less than with aztreonam and tobramycin.
Transposon mutagenesis identified few gene-inactivating mutations producing gallium resistance.

Given the unique mechanism of action of gallium, the authors investigated its combined activity with existing antibiotics in preventing $P$. aeruginosa growth. Using three independent assays - agar disc diffusion, time-kill and checkerboard - gallium was shown to exhibit synergistic interactions with colistin and the combination of piperacillin and tazobactam.

In a mouse model of $P$. aeruginosa lung infection, a single parenteral dose of gallium administered 3 or 12 hours after infection reduced lung and blood $P$. aeruginosa counts and increased survival. Notably, addition of iron solution into mouse airways immediately before infection reduced the effect of gallium, indicating that gallium's in vivo activity results from disruption of iron-dependent processes.

Given the encouraging in vivo preclinical data, the authors next tested intravenous gallium administration in patents with $\mathrm{CF}$ and chronic $P$. aeruginosa lung infections in a pilot phase Ib study involving 20 patients. A single 5-day intravenous infusion of gallium reduced sputum $P$. aeruginosa density and significantly increased lung function at 14 and 28 days, without signs of any serious adverse events.

This preliminary proof-of-concept clinical study raises the possibility that gallium may be a safe and effective treatment for human infections and further highlights the potential of therapeutically targeting nutritional vulnerabilities of bacterial pathogens. Sarah Crunkhorn

ORIGINAL ARTICLE Goss, C. H. et al. Gallium disrupts bacterial iron metabolism and has therapeutic effects in mice and humans with lung infections. Sci. Transl Med. 10, eaat7520 (2018) 